\title{
ECOS DE UNA CRISIS. HACIA LA CREACIÓN DE UN MINISTERIO DE CULTURA*
}

\author{
Pablo Chiuminatto \\ Pontificia Universidad Católica de Chile
}

\begin{abstract}
Resumen: Para el autor de esta conferencia, pese a las expectativas que genera la creación de un Ministerio de Cultura en Chile, el proyecto de ley que avanza en el Congreso y la perspectiva política que lo motiva están centrados principalmente en los creadores, los productores y cultores, considerando de forma marginal las audiencias, los espectadores, así como la relación con la formación, la circulación cultural y las economías creativas. Además, en su redacción actual, el texto presentaría graves incongruencias, asimetrías y duplicaciones en su orgánica. Aquí se arguye que el foco del proyecto es la subvención y el financiamiento de la producción cultural y artística a nivel país, con un fuerte sesgo sectorial, iden-
\end{abstract}

\footnotetext{
Pablo Chiuminatto. Artista visual. Doctor en filosofía y magíster en artes visuales por la Universidad de Chile. Profesor asociado de la Facultad de Letras de la Pontificia Universidad Católica de Chile. Entre 2012 y 2016 fue consejero, por la Región Metropolitana, del Consejo Nacional de la Cultura y las Artes. Libros recientes: René Descartes: el método de las figuras (Santiago: Orjikh Editores, 2013) y, en coautoría con Miguel Nussbaum, Diálogos para una nueva escuela en Chile: el auge de la educación (Santiago: CEPPE - Ediciones UC, 2015). Email: pchiuminatto@uc.cl.

* Versión revisada de la conferencia expuesta durante el simposio "Desafíos en el diseño del Ministerio de las Culturas", organizado por el CEP el 27 de octubre de 2016. El autor desea señalar: "Agradezco a quienes generosamente aceptaron discutir este artículo, especialmente a Pedro Gandolfo, quien estuvo a cargo de comentar la versión preliminar presentada".
} 
titario y esencialista, donde la principal figura y protagonista es el propio Ministerio de las Culturas, las Artes y el Patrimonio por venir.

Palabras Clave: cultura, institucionalidad cultural, política cultural, Estado.

\section{ECHOES OF A CRISIS. TOWARDS THE CREATION OF A CULTURE MINISTRY}

ABSTRACT: Despite the expectations surrounding the creation of a culture ministry in Chile, the author of this lecture considers that the bill now going through Congress and the political outlook that gave rise to it are heavily centred on creators, producers and proponents of culture, with only marginal consideration for audiences and spectators and for the relationship with education, cultural circulation and creative economies. As currently drafted, furthermore, the text presents serious inconsistencies, asymmetries and duplications in its composition. It is argued here that the focus of the bill is on subsidy and financing for cultural and artistic productions nationwide, with a strong sectoral, identitarian and essentialist bias, where the central figure and protagonist is the future Ministry of Cultures, the Arts and Heritage itself.

KEYwords: culture, cultural institutions, cultural policy, State.

\section{ECOS DE UN PROCESO}

E 1 Ministerio de Cultura ha tenido varias propuestas sucesivas, en[ tre el año 2012 y el 2016. La primera versión fue presentada por el ministro Luciano Cruz-Coke (en el cargo entre 2010-2013), y rediseñada luego durante el breve período del ministro Roberto Ampuero (20132014). Posteriormente, al asumir la Presidenta Michelle Bachelet, la ministra Claudia Barattini (2014-2015) encarga la redacción de un nuevo documento, luego desechado al asumir el ministro Ernesto Ottone, en 2015, quien de inmediato designa un equipo para la redacción de un nuevo proyecto. ${ }^{1}$

${ }^{1}$ Ver Pedro Bahamondes, "Ernesto Ottone, ministro de Cultura: 'Fue una señal de alerta no ver público manifestarse por el paro", diario La Tercera, 16 de enero de 2016, http://www.latercera.com/noticia/ernesto-ottone-ministro-de-cultura-fue-una-senal-de-alerta-no-ver-publico-manifestarse-por-el-paro/. 
Con los años, la discusión sobre la pertinencia de la creación de un Ministerio de Cultura se ha polarizado: todo o nada, buenos o malos. Coloquialmente formulado: a favor del ministerio o "facho". En mi caso, en principio no me opongo a la creación de un Ministerio de Cultura, con dos salvedades: según y cómo. El diseño actual es una propuesta desvinculada del Ministerio de Educación (Mineduc), que albergó al Consejo Nacional de la Cultura y las Artes (CNCA) desde su creación en 2003. Este nuevo proyecto de ley no tiene el nombre genérico de Ministerio de Cultura como los anteriores, sino que lo designa como "Ministerio de las Culturas, las Artes y el Patrimonio" (en adelante Mincap).

La discusión, sobre la necesidad de un Ministerio de Cultura, separado del de Educación, se da en la actualidad en otros países, tanto desarrollados como en vías de desarrollo. No obstante, en Chile la discusión general ha estado marcada, más que por el análisis en profundidad de sus contenidos, por un acelerado ritmo legislativo impuesto por el gobierno, con el fin de alcanzar su aprobación antes de 2018. Algunas opiniones centran como parte del objetivo de un Ministerio de Cultura, precisamente, su independencia del de Educación, mientras otros modelos los fusionan, incluso con deporte y recreación. ${ }^{2}$ En Chile la idea de mantener el actual CNCA resulta para muchos aberrante, en parte porque la creación de un ministerio autónomo es uno de los objetivos del programa del actual gobierno, en parte también porque el Mincap promete la solución de gran parte de los vacíos en las políticas culturales y en parte, cómo no, porque su creación implica un aumento importante del presupuesto anual para cultura. El problema, sin embargo, radica en que el anteproyecto y la perspectiva que lo motiva están centrados básicamente en los creadores, los productores y los cultores, y considera de forma muy marginal a las audiencias y los espectadores, así como la relación con la formación y la circulación cultural o las economías creativas. A ello hay que sumar las incongruencias, asimetrías y duplicaciones que tiene la orgánica institucional que propone. Como veremos a continuación, el foco del proyecto es el financiamiento, la subvención de la producción cultural y artística del país, con un sesgo

${ }^{2}$ Ver minuta entregada por la directora de la Dibam 2010-2014, Magdalena Krebs, a la Comisión de Educación y Cultura del Senado de la República, 14 de noviembre de 2016 . 
sectorial, identitario y esencialista, donde la figura principal y protagonista es el propio ministerio.

A priori, un Ministerio de Cultura para Chile suena como una señal positiva. No obstante, si se revisa el proceso de elaboración del proyecto de ley, además de lo registrado por la Comisión de la Cámara de Diputados y la discusión ciudadana anterior (asesores sectoriales, consejos regionales y consejo nacional), se hace evidente que el diseño actual no recoge la riqueza que aportaron las instancias participativas y el diálogo con las comunidades y los propios funcionarios de las entidades que, de acuerdo a este proyecto, serán fusionadas en el futuro Mincap. Todas estas contribuciones influyeron finalmente de forma marginal en el proyecto, salvo algunas que, por su peso político, consuetudinario o sectorial, partidista o gremial — aspectos que más adelante profundizaremos - , lograron asegurar un lugar en el organigrama. Éste, además, mantuvo aquellas instancias que ya formaban parte de la orgánica del CNCA a través de los consejos asociados: Fomento del Arte y la Industria Audiovisual ${ }^{3}$, Fomento del Libro y la Lectura ${ }^{4}$ y Fomento para la Música Nacional ${ }^{5}$, cada cual con su propio fondo concursable y vinculados, no por casualidad, a algún sector económico (fuerte) de la cultura. Por lo mismo, sectores como el teatro, las artes visuales, el folclor, la artesanía o la danza no tienen su propia línea.

La Presidenta Bachelet presentó la "Indicación sustitutiva al Proyecto de Ministerio de Cultura" el día 17 diciembre de 2015. Pasó así a la Cámara de Diputados, disponiéndose una comisión a cargo. ${ }^{6} \mathrm{La}$ publicación que podría haber marcado un auspicioso umbral, sin embargo, sufrió un primer traspié. Los cambios realizados al proyecto de forma unilateral por el gobierno, horas antes de hacerlo público, motivaron el paro de la Asociación Nacional de Funcionarios (Anfudibam) de la Dirección de Bibliotecas Archivos y Museos (Dibam). La movilización

\footnotetext{
${ }^{3}$ Ver Ley 19.981.

${ }^{4}$ Ver Ley 19.227.

${ }^{5}$ Ver Ley 19.928.

${ }^{6}$ Comisión de Cultura, Arte y Comunicaciones, integrada por los diputados: Ramón Farías (PPD y presidente de la comisión), Claudio Arriagada (DC), Marcos Espinoza (PRSD), Maya Fernández (PS), Joaquín Godoy (I), María José Hoffmann (UDI), José Antonio Kast (hoy ex UDI), Issa Kort (UDI), Roberto Poblete (I), Jorge Rathgeb (RN), Gaspar Rivas (I), Guillermo Teillier (PC), Víctor Torres (DC). Ver https://www.camara.cl/trabajamos/comision_portada.aspx?prmID=496.
} 
durará cerca de un mes. El diseño del ministerio, con dos subsecretarías y la Dibam y el Consejo de Monumentos Nacionales (en adelante $\mathrm{CMN}$ ) convertidos en "servicios", genera dos categorías funcionarias. ${ }^{7}$

Estas diferencias aparentemente formales del organigrama, y por lo tanto de la orgánica, son las que, tal como veremos en la sección 5 de este ensayo, es preciso que sean corregidas en un nuevo proyecto de ministerio, ya que, además de fusionar estamentos preexistentes, no hace sino replicar — casi sin diferencias - el actual CNCA. De paso, el diseño propuesto desnaturaliza instituciones históricas como la Dibam y el CMN, convirtiéndolos en servicios públicos. ${ }^{8}$ La Dibam con casi un siglo de existencia, en vez de verse potenciada en el nuevo ministerio, es reducida a su mínima expresión, mientras que el recientemente creado CNCA pasa a dominar la estructura. Es decir, luego de todo el esfuerzo que representa el estudio y el diseño para la creación de una nueva institucionalidad cultural, se establece un ministerio con dos visiones de desarrollo del Estado en una misma entidad: un área de "las culturas y las artes" perteneciente al ministerio y "el patrimonio" reducido a servicio, decisión que implica procesos muy distintos de ejecución de la política pública, volviéndolo un diseño, a la larga, inviable que seguramente, como otras reformas implementadas durante el gobierno de la Nueva Mayoría, deberá reorganizarse al poco andar.

El término del paro de la Dibam, a fines de enero de 2016, permitió que el CNCA pueda centrarse en las sesiones de consulta de la Comisión de la Cámara. Allí se dio un importante debate, pero en general, salvo excepciones, los cambios al proyecto fueron menores. Cada vez que se tocaba el fondo del diseño o la estructura que lo sostiene se apeló al artículo 65, inciso cuarto, número $2^{\circ}$ de la Constitución Política de la República, que reserva los cambios al diseño presentado como materia exclusiva de iniciativa presidencial.

7 Ver minuta presentada por Margarita Hormazábal, de la Anfudibam, y Francisco Silva, en representación de los funcionarios del CMN, ante la honorable Comisión de Educación y Cultura del Senado, 16 de noviembre de 2016. Los funcionarios del CNCA (Anfucultura) quedan comparativamente en mejor pie, porque, entre otras diferencias, pasarán de una planta básicamente a honorarios a una con contrato indefinido. Una diferencia aparentemente formal, pero que refleja tramos distintos para la carrera funcionaria, igualados para efectos de la negociación y fin del paro de la Dibam, pero que en su futura definición estructural serán distintos.

${ }^{8}$ Ver el organigrama incluido en el Boletín 8938-24, 82. 
Mientras ese eje continuó su desarrollo y una vez que el paro de los funcionarios terminó, se acercaba el receso legislativo. Para bajar la presión pública y de los medios, la propia Comisión de la Cámara se comprometió a convocar a una jornada amplia de trabajo. La fecha se fijó cuando enero terminaba y se invitó y publicitó sólo una semana antes. Fue el día 7 de marzo de 2016, bajo el nombre de jornada temática "Creación Ministerio de Cultura y nueva institucionalidad". Los invitados fueron seleccionados por la comisión y el propio CNCA e incluyó un discurso final del ministro Ottone. La dinámica de las presentaciones fue sucedida por rondas de preguntas del público y de los diputados integrantes. El resumen de esa jornada se puede revisar en el informe final de la Comisión. ${ }^{9}$ Sin embargo, ese documento no recoge ninguno de los comentarios ni de las preguntas del público. Tampoco el discurso que hace el ministro Ottone al cierre. Pero este sesgo no impresionó a nadie, puesto que es ése el concepto de participación ciudadana con que opera el CNCA y sus órganos colegiados: cuando sirve, se apela a la participación; cuando no, se define verticalmente ya sea por el director regional, el ministro o el Presidente de la República. Esta capacidad operativa se acentúa aún más en el proyecto actual, tal como veremos en la sección 5 de este artículo.

El proceso continuó y el ejecutivo se impacientaba ante las sesiones semanales en la Cámara, que se sucedían sin el resultado esperado y su paso a votación. Es así como los primeros días de mayo de 2016 el gobierno le puso "suma urgencia" al proyecto. ${ }^{10}$ Todo se acelera. Esto, por otra parte, asegura la suspensión de las audiencias en las que se escucha a las partes y pone un foco prioritario para su paso al Senado. El día martes 2 de agosto de 2016 el pleno de la Cámara aprobó el anteproyecto con 109 votos (de 110) y una abstención. Fueron planteados algunos comentarios en la sesión, pero la propuesta fue refrendada sin observaciones importantes. ${ }^{11} \mathrm{Si}$ bien para muchos la votación hace patente que Chile necesita un Ministerio de Cultura y que la mayoría lo

\footnotetext{
${ }^{9}$ Boletín 8938-24, 94-110.

${ }^{10}$ Comisión de Cultura, Arte y Comunicaciones, 3 de mayo de 2016, Boletín 8938-24.

${ }^{11}$ Cámara de Diputados, legislatura $364^{\mathrm{a}}$, sesión $48^{\mathrm{a}}$, 2 de agosto de 2016. Es importante reconocer que los únicos en manifestar aprensiones relevantes al texto en su estado actual, desde distintas perspectivas, fueron los diputados Issa Kort (UDI), Gabriel Boric (MA) y Giorgio Jackson (RD).
} 
apoya, mi perspectiva es que esta elocuente aprobación refleja la utilidad que tendría para todo gobierno este proyecto de ley, cualquiera sea la línea política. Esta es la mejor prueba.

En consecuencia, el proyecto pasó a revisión a la Comisión de Educación y Cultura del Senado. ${ }^{12}$ Esta comisión abrió un nuevo ciclo de entrevistas y presentaciones de personas, instituciones y representantes (20 en total); así como de invitados del CNCA o el propio ministro o su representante en la sala. Esta fase debe continuar hasta que la comisión senatorial decida y pase a votación en sala o vaya a Comisión Mixta, donde la discusión debe recomenzar. ${ }^{13}$ El cambio de una sola coma, letra o acápite del proyecto actual es causa suficiente para que tenga que ser revisado y discutido por una comisión compuesta por senadores y diputados. Si así ocurre, sería necesario que el gobierno reconociera, por una parte, que fue el dogmatismo y el secretismo del proceso lo que motivó las críticas que cosechó. Y, por otra, debiera reconocer también las graves asimetrías e incongruencias en la redacción final del texto, así como el organigrama derivado. Si el objetivo era establecer un Ministerio de Cultura que en la práctica será una red asistencial a la creación y a la producción cultural, el gobierno debió haber cuidado el aspecto participativo real. Como dije al inicio, el discurso que antecedía a la "Indicación sustitutiva" ya anunciaba ese esencialismo radical, su marca identitaria y chovinista, lo que tiñe el proyecto de ley desde sus cimientos.

\section{ECOS DE UNA DISCUSIÓN}

Lo que más me impresionó de la lectura del texto desde su primera presentación como "Indicación sustitutiva", en 2015, fue que tanto su redacción como su alcance de fondo instalaban un marco cultural, legal y político basado en un objetivo mayor: la apropiación estatal sesgada -ideológica e históricamente- de la cultura en Chile. Quiero decir con esto que, más que un diseño pensado para establecer y planificar

${ }^{12}$ La Comisión de Educación y Cultura del Senado de la República es constituida por los honorables senadores: Andrés Allamand (RN), Jaime Quintana (PPD), Ignacio Walker (DC), Ena von Baer (UDI) y Carlos Montes (PS).

${ }^{13} \mathrm{Al}$ cierre de esta edición de Estudios Públicos, 15 de marzo de 2017, el proyecto permanecía en discusión en el Senado. 
políticas culturales, lo que hace el proyecto es instituir un programa cultural como marco político nacional, donde pareciera que no hay cultura sin Estado.

Es más, si se revisa el discurso que antecede la "Indicación sustitutiva", se aprecia que lo que está en el texto no se hace en nombre de la cultura, sino de una "verdad cultural" mediada por la adjudicación de instancias particulares de justicia histórica: para los artistas, para los cultores, para los creadores, para los indígenas, para los sectores productivos del libro nacional, para la música nacional y para el campo audiovisual nacional. Chovinismo aparte, se produce una equivalencia entre financiamiento cultural y justicia histórica. Por otra parte, como ya expliqué, el texto olvida de forma patente a las audiencias y los espectadores (más en la sección 5). Y tal como en educación, en el caso de cultura se impone un currículum centralizado y nacional. Las consecuencias están a la vista.

Como señalé, el Estado se vuelve el protagonista de la cultura. Este rasgo podría no ser malo en sí mismo, si efectivamente el documento del proyecto obedeciera a un diseño renovado, flexible y dinámico, que considerara la versatilidad del campo cultural en el nuevo contexto global. Por el contrario, el diseño del Mincap obedece no sólo a una retórica cultural, identitaria, nacionalista, memorialista y, del mismo modo, homogeneizante de las diferencias y singularidades de los contextos locales; sino que, además, establece estructuras asimétricas para los tres ejes nominales que complementan su designación: las culturas, las artes y el patrimonio. La solución, contradictoria, es nominal, es textual, queda en el texto como aparentemente inclusiva, diversa y multicultural. No apuesta por un espacio de encuentro en la diversidad, sino de diferencia en la diferencia. ${ }^{14}$ Es decir, cuando por fin se alcanza la creación de una entidad mayor que vele por las políticas culturales a nivel nacional, lo que hace la propuesta del gobierno es fragmentar lo que podría unir, además de alterar el equilibrio de las partes que componen históricamente el ecosistema de la cultura nacional. En resumen, el diseño de ministerio lo que hace es replicar el CNCA, agregar el CMN y reducir al mínimo a la Dibam, que es la que efectivamente posee la red cultural más amplia a nivel nacional a través del sistema de bibliotecas públicas,

${ }^{14}$ Ver carta de Marta Cruz-Coke (directora de Dibam 1993-2000) en diario El Mercurio, 23 de noviembre de 2016. 
después del Mineduc y su red nacional educacional. Todo esto, además, en nombre de una esperada justicia cultural para Chile.

Ahora bien, el concepto de nación le ha costado siempre a Chile. Cualquier forma de identidad es rechazada por esencialista, salvo el esencialismo estratégico que asume el Estado cuando se trata de incorporar a las comunidades como capital político. En este contexto de diseño del Mincap, el discurso identitario, por ejemplo, de los pueblos indígenas, de los gremios o de las denominaciones sectoriales tradicionales sirven para un bien superior, la ampliación del concepto de Estado, pero que, no obstante, el gobierno intenta incautar para sí.

Este caso no es distinto. Básicamente, el proyecto del Mincap opera bajo una lógica de facción, salvo por una omisión importante, el olvido de la consciencia de cambio, de cambio de mano del poder. Dicho en otras palabras, no se trata de un diseño institucional pensado para el futuro de la cultura nacional - que considere el efecto pendular del poder-, sino que se constituye bajo la ilusión de que esta entidad del Estado se mantendrá en una misma línea de poder. Es decir, se olvida aquello que bien anuncia Hegel cuando señala que "la facción triunfante se llama gobierno y justamente en ello, en el ser una facción, radica de modo inmediato la necesidad de su perecer". ${ }^{15}$ Es este antiguo mensaje el que el gobierno de la Nueva Mayoría olvida. Basta hacer un poco de ficción: ¿cuál sería su opinión de este mismo proyecto en manos de otro gobierno, de otra fuerza política?

El esencialismo del proyecto que presentó el CNCA no sólo tiene que ver con que incorpore de forma nominal a los indígenas, sino que también lo hace respecto de las técnicas, los dispositivos y los medios relacionados con las artes y la cultura, igualándolos. Es un extraño efecto totalizante de entidades esenciales.

Aunque no profundizaré aquí sobre los problemas que enfrenta al Estado con los pueblos indígenas, es evidente que aquellos relacionados con cultura no son los más relevantes, como sí lo son los recursos naturales, ambientales y territoriales. Sin desconocer, por cierto, los compromisos internacionales adquiridos con el acuerdo de la OIT (Convenio 169) y la Unesco, tales acuerdos no son suficientes para fundar de

${ }^{15}$ G. F. W. Hegel, Fenomenología del espiritu, citado en Martín Cerda, "El espíritu faccioso", en Escombros: apuntes sobre literatura y otros asuntos (Santiago: Ediciones Universidad Diego Portales, 2008), 68. 
manera esencialista desde el diseño hasta el nombre del futuro ministerio. Lo justo sería entonces que cambiáramos los nombres de otros ministerios: ministerio de las economías, las justicias y las educaciones, sólo para dar un ejemplo.

Broma aparte, desde mi punto de vista la jugada retórica, demagógica, de configurar el Ministerio de las Culturas, con esos plurales en atención a la existencia de pueblos originarios, tiene efectos políticos claros e implica un peligro y una limitación. Se está creando un organismo operativo del Estado, no estableciendo un acto de reparación histórica. Es una institución que debe funcionar de forma estable en el futuro. Se trata de una aparente corrección nominal en una nación singular que operará bajo una única legalidad, sin el reconocimiento de otras naciones en su interior. Jugar al reconocimiento, cuando no es verdadero, es serio.

Ficciones identitarias, cuando lo que necesita Chile son prácticas igualitarias. A eso se suma un fantasma aún mayor, el de la posibilidad de acotar el mestizaje, la mezcla que es patente y que está en constante asimilación. Éste es un punto álgido del diseño general, el de la pureza de linaje, tanto de los pueblos como de los gremios, de las áreas, de las prácticas, de los sectores productivos, así como de las industrias culturales, todo desde una perspectiva esencial. Así lo evidencia el diseño de la orgánica del Mincap: designaciones genéticas complejas que, cuando son utilizadas por el Estado como forma de reparación y reconocimiento, se vuelven al mismo tiempo instrumentos de asimilación y asentimiento político. Manipulación ontológica tras un discurso de tolerancia e integración. ¿Qué se viene en cultura, procesos ius soli, ius sanguinis?

La insistencia del CNCA en transformarse en el articulador principal o mediador de la relación con los pueblos indígenas, aunque sea bien intencionada, es instrumental y, tal como se ve en la prensa en los últimos años, no alcanza el resultado esperado por el gobierno. El Estado insiste en focalizar la solución en "gestos" que, a pesar de ser importantes en la relación, implican un nivel de negociación muy distinto de los conflictos relacionados con cuestiones históricas, pero también territoriales, en un desencuentro que simultáneamente mella otro principio que el CNCA trata de monopolizar: el de la "memoria". Me pregunto en qué momento se separaron, en qué momento se volvieron dos cosas tan 
distintas tradición y memoria, casi como formas opuestas. ${ }^{16}$ ¿Cómo es que Chile llegó a un modelo donde la cultura se confunde con el Estado y donde más que considerar a las comunidades de referencia efectiva, se busca la identificación con el aparato estatal que la gestiona? Por eso es necesario corregir desde el diseño mismo esta deformación, por medio de la integración de las políticas culturales a procesos mayores, como la superación de la desigualdad, las brechas de participación cultural y de educación. ${ }^{17}$ Porque, aunque este proyecto no lo considere, existe una cultura sin Estado. ${ }^{18}$

Hoy por hoy, el concepto de "cultura nacional", de algún modo, es un oxímoron. Entre Violeta Parra y Daddy Yankee, la cultura no tiene un adentro y un afuera. El préstamo cultural internacional, transnacional, no se detiene ante la formalización administrativa. La noción de un CNCA y su supuesto proceso natural hacia un ministerio, derivado de su constitución burocrática, alimentan la ficción de una unidad que lo local y plural vuelve impracticable. Sobre todo cuando lo nacional, en un sentido amplio, está en crisis; cuando las comunidades locales y las agrupaciones espontáneas y circunstanciales empiezan a presionar los límites desfigurados de los partidos políticos y las ideologías. En este sentido, la valencia de subsidiariedad del proyecto está marcada por la idea de darle a todos un poco. Por eso se plantea la división por sectores productivos en una rama del organigrama ministerial y, en la otra, se definen identidades étnicas, técnicas o dispositivos mediales (ver sección 5, g). Curiosa la mezcla, si al otro lado del organigrama van las bibliotecas públicas y el Consejo de Monumentos Nacionales (ver 5, h), categorías que mezclan el hacer con el saber y con el ser.

\section{ECOS IDENTITARIOS}

En Historia de las ideas y de la cultura en Chile, Bernardo Subercaseaux hace presente la necesidad de distinguir tiempos diversos para Chile: un tiempo fundacional, uno de integración, uno de transformación

${ }^{16}$ Ver Beatriz Sarlo, Tiempo pasado: cultura de la memoria y giro subjetivo. Una discusión (Talca: Editorial Universidad de Talca, 2013).

${ }^{17}$ Ver Bárbara Negrón, “El 'estado del arte’ en Chile”, Revista Periférica Internacional 15 (2014): 182.

${ }^{18}$ Ver Karine Berger, Manuel Alduy y Caroline Le Moign, La Culture sans État: de Modiano à Google (Paris: Odile Jacob, 2016). 
y uno globalizado. ${ }^{19} \mathrm{Si}$ bien estoy de acuerdo con la propuesta del autor, ante el proyecto del Mincap, resulta crucial plantear una interpretación desplazada de estos conceptos. Es decir, respecto de las políticas culturales, los "tiempos" de Chile debieran pensarse abiertos y dinámicos, paralelos, no excluyentes y, mucho menos, progresivos. Porque dichos tiempos conviven en el territorio y no se han cerrado como si se tratara de eras precisas. Chile vive temporalidades paralelas con comunidades, lugares y grupos humanos en simultaneidad de eras: comunidades que aún viven en un tiempo fundacional; territorios en los que la integración y la transformación coexisten; estratos sociales que tienen acceso al tiempo globalizado y otros no. El mestizaje no se detiene, las migraciones tampoco, incluso la fundación o refundación puede entenderse como un fenómeno permanente. Las instituciones nacionales de igual forma, la cultura también. ${ }^{20}$ Quizás sería más coherente pensar en una institucionalidad que tendiera hacia lo posnacional o bajo criterios de desnacionalización, dada la experiencia de otros países integrados por comunidades y grupos étnicos diversos. ${ }^{21}$ Sin embargo, lo que primará en Chile, si se aprueba el Mincap tal como está ahora, serán planes y programas nacionales, con una confusa expresión en la ley, con decenas de direcciones regionales a partir de los servicios públicos establecidos, sin real autonomía de los territorios, sin una apropiación verdadera de la política pública (ver sección 5, f). El control de esas "culturas" en plural será central.

La cultura en Chile resulta un tema poco relevante si no se comparte la percepción que la vincula y explica como parte de la crisis de

${ }^{19}$ Bernardo Subercaseaux, Historia de las ideas y de la cultura en Chile. El centenario y las vanguardias (Santiago, Editorial Universitaria, 2004), 13: "El tiempo fundacional a comienzos del siglo XIX, desde el período de la Independencia hasta la crisis en la década final del siglo; el tiempo de integración, desde fines del siglo XIX hasta las primeras décadas del XX; el tiempo de transformación, desde la década del treinta hasta la década del setenta, y, finalmente, el tiempo globalizado, entre 1980 y el presente".

${ }^{20}$ Una propuesta similar, pero dirigida a comprender la situación de las artes visuales, hace Justo Pastor Mellado en su presentación en la primera jornada para la creación de la política de artes visuales del CNCA, "Artes de la visualidad y política de Estado", el 21 de julio de 2016. Ver: http://www.cultura.gob.cl/wp-content/ uploads/2016/08/Mellado_Escenas-Locales_.pdf.

${ }^{21}$ Ver Saskia Sassen, "Incompletitud y la posibilidad de hacer. ¿Hacia una ciudadanía desnacionalizada?”, Revista Mexicana de Ciencias Politicas y Sociales 61, n. ${ }^{\circ} 226$ (2016): 107-139. 
un ecosistema - tanto de educación, de representatividad política y económica-, como un factor integrado que trasciende a todas las dimensiones de la sociedad. La crisis de Chile podrá entenderse cuando sea posible comprender su matriz cultural. No basta decir que la cultura es diversa, que hay varias, porque a esta altura parece hasta frívolo. El proyecto recurre a las comunidades, pueblos, grupos y gremios y los reconoce en su diferencia, pero luego los empaqueta para consignarlos en el concepto nacional que todo ministerio implica. De ahí no podrán volver a salir. ${ }^{22}$ Sé que parece sólo una cuestión conceptual, pero cuando se trata de leyes, eso cuenta. Al leer a fondo el texto del proyecto queda en evidencia que el gobierno buscaba, como señalé antes, entre otros objetivos, reapropiarse de una huella histórica precisa, por medio de la instalación de una valencia simbólica, para que el proyecto fuera identificado perpetuamente con el sesgo de la coalición de izquierda y centro que lo diseñó. Un sello donde la cultura debe ser subvencionada, en un sentido reparatorio, no sólo en la acepción del gesto hacia las víctimas de un pasado antidemocrático, sino abarcando ahora un espectro mayor, amplio, "la justicia cultural", terreno en el que, si se revisa el discurso de la Presidenta de la República en la presentación de la "Indicación sustitutiva 2015", todos los sectores, incluido el Estado mismo, aparecen como una víctima, un postergado, una minoría.

Un Estado víctima que requiere de esta justicia cultural: una figura muy especial. De ahí la influencia en los textos y en las discusiones públicas donde, a pesar de décadas de gobiernos democráticos, lo que se busca es el equilibrio de culpas y traumas. Donde el Golpe Militar de 1973 y los atropellos de los derechos humanos durante la dictadura de Augusto Pinochet (1973-1990), los asesinatos y las desapariciones se vuelven un tópico central de la negociación simbólica y política detrás del diseño del Mincap. Y si esto ya es una carga importante para el documento, es preciso sumar además la deuda para con los pueblos indígenas.

Ahora bien, la pregunta es: ¿Por qué el Ministerio de Cultura es el que debe hacerse cargo de esta justicia cultural e histórica que el mismo Estado no ha sido capaz de darse en casi treinta años de de-

${ }^{22}$ Ver Saskia Sassen, “¿Globalización o desnacionalización?”, en Viejas sociedades, nueva sociología, editado por Juan Monreal, Capitolina Díaz y J. J. García Escribano (Madrid: Centro de Investigaciones Sociológicas, 2005), 65-86. 
mocracia? ¿Por qué el propio Estado, a pesar de todo lo que ha hecho y los recursos que ha aportado, mantendría una deuda con la cultura? Deudas no muy distintas a las que hoy se escuchan en la calle en salud, pensiones, educación y energía. Si se revisan los documentos y las discusiones en cultura, no sólo resurgen las consecuencias de los conflictos políticos e ideológicos de fines del siglo XX en Chile, sino que además, de algún modo, se refleja en la misma lógica estructural del texto, pero de forma anacrónica y expandida a toda la historia de Chile. Es un lema de fondo: "Chile me debe". Por esta razón me parece que este proyecto de ley es una propuesta historiográfico-programática que rearticula lo nacional antes de la nación; una institución cultural que hará justicia respecto del Estado mismo en cuanto víctima, desde antes de que Chile fuera Chile.

La perspectiva historiográfica que este diseño de ministerio busca instalar tiene un efecto retrospectivo interesante. Construye la ilusión anacrónica de atribuir todas las culpas - las actuales y las antiguas - a los ideales políticos del bando contrario, en tanto que la alternativa correcta son los ideales políticos propios, reduciendo la discusión a una cuestión entre progresistas y conservadores, buenos y malos. De lo simbólico a lo concreto, de lo real a lo cultural, una polaridad que pareciera insuperable en Chile: izquierda y derecha. Por eso pienso que es necesario aspirar a un nuevo proyecto de ley que comprenda lo que Daniel Mansuy describe al inicio de su reciente libro:

... si acaso deseamos comprender nuestra crisis, debemos distanciarnos de las disposiciones reflejas, que ven en toda concesión o acuerdo un signo de derrota política; o que conceden demasiado buscando simplemente acomodarse a la opinión dominante. No se trata de buscar la quimera de la neutralidad pura (que, sobra decir, no existe en estos asuntos), sino de tomar alguna distancia respecto de las aproximaciones partisanas, que persiguen anotarse un triunfo táctico antes de comprender y reflexionar. ${ }^{23}$

El "esfuerzo comprensivo", lo expresa Mansuy, sin duda es urgente. La vuelta a la deuda eterna provocada por el quiebre antidemocrático y sus procedimientos brutales y sistemáticos, cuarenta años después,

${ }^{23}$ Daniel Mansuy, Nos fuimos quedando en silencio (Santiago: Instituto de Estudios de la Sociedad, 2016), 15. 
aún no encuentra sosiego. Quizás, de acuerdo a lo que expresan los propios expertos en derechos humanos, porque no ha pasado suficiente tiempo. Es en este momento cuando surgen para mí otras preguntas que, por cierto, no tienen fácil respuesta: ¿Es el Ministerio de Cultura un Ministerio de la Memoria, un Ministerio de la Compensación Histórica? ¿Es eso lo que la ciudadanía necesita urgentemente?

La motivación para haber diseñado este proyecto de ministerio con un sesgo tan marcado es simple: se trata de razones políticas, de política. No obstante, cuando se discute cada punto de la creación del Mincap, lo que se pone en escena no es esa fuerza mayor. Por lo mismo, la aprobación del Mincap es algo más que simplemente su consenso o reprobación en cuanto ley, sino la posibilidad de sellar simbólicamente la formalización institucional convertida en un proyecto histórico de izquierda. En este ministerio, tal como lo demuestran los documentos, se está tratando de hacer justicia histórica, justicia social, justicia indígena y justicia en la participación ciudadana. ¿Es eso un Ministerio de Cultura? Quizás sí, por lo poco relevante que aparece para muchos el Ministerio de Cultura y, al mismo tiempo, por lo relevante que será como aparato de propaganda para los gobiernos que vendrán. El fantasma del populismo que hoy por hoy emerge en tantos análisis está presente en este cruce entre rasgos demagógicos y oportunistas de participación ciudadana, y de articulación de un supuesto deber o deuda - en ese sentido amplio que mencionaba - con esa misma ciudadanía que se siente adeudada (cuestión sobre la que me extenderé a continuación).

\section{ECOS DE LA PARTICIPACIÓN}

En la jornada de diálogo que organizó la propia comisión de la Cámara de Diputados, el 7 de marzo de 2016, en el ex Congreso Nacional, hubo una intervención que refleja con claridad el tipo de dudas que los ciudadanos tienen ante la creación del Mincap. Sólo a modo de ejemplo, en el acápite "pueblos originarios", el documento de la Cámara recoge lo que Juan Carlos Tonko planteó:

... advierte sobre el alcance amplio que tienen las nociones de cultura y patrimonio para su comunidad kaweshkar, señalando 
que la autodeterminación, el territorio, la cultura y el patrimonio son un continuo que no se puede separar. Asimismo, expresa su preocupación frente a lo que él considera el enfoque sectorial que está adquiriendo la iniciativa tratada, toda vez que en "cultura" comprende sus tradiciones, lengua, conocimiento tradicional y estilos de vida, pero también incluye una base material, que para su pueblo está representada en el acceso y uso de sus recursos y territorio. $^{24}$

Este pasaje deja claro el peligroso juego que esconde el velo demagógico de la multiculturalidad con que avanza este proyecto de ministerio. Obviamente, nadie podrá acceder a la autodeterminación a la que aspira Tonko, porque un ministerio de cultura es nacional y es de la cultura de un país, y las propias nociones de territorio que las comunidades se den no está en proceso de revisión. Por el contrario, no serán sino parte del proceso de negociación que cruza el paradigma gobierno-Estado, administrando el capital simbólico y material que el futuro ministerio obtendrá como trofeo.

Es en este rango de posibilidades donde se alza el otro fantasma que tiene sobre sí todo Ministerio de Cultura, cuando se centra en un proyecto historiográfico en sí mismo. Un ministerio, hoy CNCA, que mutó hacia una entidad de programación centralizada y de asistencia a la creación artística, cuando, si se hubiera querido, se podría haber diseñado uno enfocado en la planificación cultural abierta a la participación en un sentido amplio; un ministerio más centrado en impulsar las prácticas culturales a través de la promoción del cultivo de lo que las propias comunidades identifican como referencias culturales para sí mismas. La noción de "acceso" a la cultura como principio primó por años, sin embargo, poco a poco fue mostrando sus falencias, dada la variedad en la calidad y la improvisación que representa un sistema mixto de intervención cultural como el que tiene el CNCA: una "parrilla programática" con planes y programas propios pero, además, con la carga de cientos de proyectos realizados por medio de los fondos concursables. A partir de esta experiencia, era esperable un cambio en

24 "Informe de la Comisión de Cultura, Artes y Comunicaciones recaído en el proyecto de ley que crea el Ministerio de Cultura”, Cámara de Diputados, 14 de junio de 2016, Boletín 8938-24, 96. 
la gestión de esos planes y programas así como de los fondos. Pero no fue así. Considerando que si se buscaba ayudar a los ciudadanos más alejados de los estratos medios y altos, ello no se logra sin una base seria de estudios centrados en las audiencias y los espectadores; es decir, en saber qué podría querer o necesitar la ciudadanía, más allá de la verticalidad exclusiva y excluyente de la cultura entendida como lo que se le da al que no tiene acceso. A los fondos concursables, en cambio, paradójicamente acceden los mismos que tienen acceso a la mejor educación. Es decir, el concepto de acceso opera para los pobres, para que puedan exponerse a la cultura, tener contacto con ella (nuevamente un rasgo esencialista, como si la cultura fuera algo, una cosa y no un todo indivisible). La ecuación entonces quedó configurada así: programación y acceso para los mismos que reciben una educación que fracasa en el 70 por ciento y proyectos y fondos para los que reciben el 30 por ciento de la buena educación de Chile.

La cita que recojo resume en parte la presentación de Juan Carlos Tonko en aquella jornada y revela otro aspecto a considerar, anexo al de las identidades: es el concepto mismo de participación que este proyecto de Mincap hereda del CNCA. En su transferencia al modelo de ministerio la participación muta hacia conceptos más grandilocuentes, aunque sólo con el rol de opinar y recomendar, no vinculante en sus decisiones.

No en vano esto vuelve a aparecer en una primera pregunta que hace Ramón Farías, diputado miembro de la Comisión, en una de las tantas sesiones durante la discusión del proyecto, a propósito de los consejos ciudadanos y participativos. Su consulta no estaba dirigida a cómo el proyecto ayudará a los más desposeídos, ni a los pueblos indígenas, ni a los ancianos, ni a las personas con discapacidad, ni a los niños de Chile. Su pregunta era otra. Cito al diputado Farías:

¿Cómo se resuelve el problema de que el día de mañana cambia un gobierno y el(la) Presidente(a) de la República nombra a un ministro(a) de las culturas y desea llevar adelante una política cultural comprometida al país en su programa, y no pueda porque resulta que existirá un Consejo Nacional de las Culturas y las Artes y el Patrimonio que definirá las políticas y no el ministro? ${ }^{25}$

${ }^{25}$ Ibídem, 91. 
El ministro Ernesto Ottone, que estaba presente, respondió citando el artículo 2 , inciso $1^{\circ}$ del proyecto. La respuesta satisface al diputado, porque en dicho acápite se precisa que la participación de los consejos civiles será parcial y estará bajo el control del ministro o los subsecretarios respectivos, y que ningún consejo, tanto nacional como regional - tenga la conformación numérica que tenga-, podrá quitarles la autonomía y predominio a los objetivos de la Presidencia de la República.

Lo más grave es que en el proyecto la redacción de la descripción y explicación de la conformación de los consejos participativos ocupa gran parte de su extensión, aunque si se analiza en profundidad su alcance quedan claras las limitaciones y, sobre todo, el hecho de que están bajo la dinámica de un teatro democrático - estoy siendo irónico y no metafórico- , donde el presidencialismo se deja ver en todo su esplendor (ver sección 5, j). Consejos regionales y uno nacional compuestos, entre otros, por una diversidad indefinida que el proyecto señala como integrantes: representantes de las "industrias culturales", las "culturas tradicionales", las "culturas populares", las "culturas territoriales", entre otras etiquetas. ${ }^{26}$ Prácticamente todos, además, serán elegidos por el propio ministro(a) para una verdadera participación no vinculante, es decir, falsa. ¿Por qué no llamarlos directamente comités consultivos y no consejos?

Las culturas, las artes... pero, sobre todo, un ministerio. Uno para varias culturas. Con esto se hace evidente que la propuesta, en su articulación orgánica en tres áreas separadas (institucionalidad, fondos y patrimonio) tiene objetivos y estrategias diversas que no siempre deberán o podrán estar alineadas. En el caso de los fondos concursables, éstos generan toda una articulación respecto de las áreas de producción, lo que no siempre estará en directa proporción respecto de la difusión, la educación o la circulación del arte y la cultura. Menos aún respecto de las necesidades de los más necesitados. Aunque bajo la denominación de "necesitado" no estoy refiriéndome sólo a los que tienen menos acceso al dinero y al poder. Es claro en la crisis actual que vive Chile que también es necesario, y quizás igualmente urgente, preocuparnos de la educación y la cultura de las clases dominantes. En este sentido, sin

${ }^{26}$ Ver Boletín 8938-24, 231 y ss. Una perspectiva aguda de estas imprecisiones conceptuales fue señalada por el periodista Álvaro Matus en "Todas las voces todas", diario La Tercera, 8 de diciembre de 2016, http://www.latercera.com/voces/ todas-las-voces-todas/. 
mayor profundidad, apenas se mencionan las industrias creativas, pero tampoco se asume las implicancias que tienen el propio Estado y los fondos concursables sobre el mercado del arte (en un sentido amplio). No existe así, en esta futura plataforma ministerial, tanto al servicio de los artistas y cultores como de los espectadores y audiencias, una integración de ambas partes en la misma proporción, ${ }^{27}$ ya no en términos identitarios, sectoriales o gremiales solamente de igualdad real, no terminológica.

Esta institucionalidad además sumó otro aspecto capitalizable por el Estado: "la cultura como derecho". ¿Qué puede significar esto? Será un derecho como el derecho a la educación y a la salud, que como es bien sabido no logran estándares satisfactorios en Chile. ¿Escolarizarán la cultura? ¿Se establecerán planes y programas bajo una edición de contenidos que permitan un currículum nacional en cultura, como en el caso de la educación nacional? Resulta, por decir lo menos, paradójico: es precisamente este proyecto de ministerio el que separa educación y cultura prácticamente en todas las instancias en que se supone que puede prescindir un área de la otra. En fin, vayamos al texto mismo del proyecto.

\section{ECOS DEL TEXTO28}

Las disputas ideológicas que evidencia el documento final, incluida la discusión de las comisiones, hace patente lo que he planteado en términos generales. Lamentablemente, la dinámica con que ha operado el gobierno no busca un debate público real, sino que apela a la sensibilidad pública.

Ahora señalaré de forma específica, en el texto del proyecto de ley, algunos de los puntos que fundamentan lo que antes he mencionado. Tal como subrayé antes, la votación de la Cámara de Diputados fue de 109 votos a favor (de 110) y una abstención. Esto demuestra cómo no sólo la izquierda o el centro, sino también la derecha ve con buenos

\footnotetext{
${ }^{27}$ Ver Carlos Javier Villaseñor Anaya, "Retos para la gestión cultural, en América Latina", Revista Periférica Internacional 15 (2014): 262-266.

${ }^{28}$ Una versión preliminar del texto que compone esta sección la redacté para la presentación que hice ante la Comisión de Educación y Cultura del Senado, el 16 de noviembre de 2016.
} 
ojos lo que instala este diseño. Mi esperanza es que la Comisión de Educación y Cultura del Senado exija una comisión mixta o, también, que simplemente el proyecto sea abandonado por el gobierno, dado que podría volverse un lastre innecesario en este último año de gestión.

a) En "Principios" (art. 1): Dada la naturaleza propia de los principios debiesen ofrecer definiciones y descripciones adecuadas, de manera de facilitar la comprensión del objetivo que se busca, cuestión que en el texto no se hace. Además, en este punto, queda en evidencia la accesoria consideración de audiencias y espectadores, así como de mediadores culturales. Pareciera que más que un ministerio de cultura asistiéramos a la creación de una red asistencial para los creadores y la conservación, en desmedro de otros estamentos de alcance nacional como las escuelas, los liceos y las bibliotecas públicas de Chile. El Mineduc tiene una relación directa con los docentes que integran el sistema nacional de educación, sin embargo, este Ministerio de Cultura pareciera no estar concebido bajo un paradigma equivalente, desaprovechando el potencial de integración, capacitación y profesionalización para la mediación cultural. Por lo anterior, es fundamental que en los "Principios" quede establecido uno particular para este ámbito: audiencias y espectadores.

b) En "Funciones y atribuciones" (art. 3): Los verbos rectores son débiles. Paradójicamente, resalta la imprecisión en las áreas de fomento y creación, comparada con las minuciosidades en las que se adentra el articulado cuando define las instituciones patrimoniales (Art. 29), cuando son ellas las que poseen un bagaje histórico, centenario - museos nacionales, Biblioteca Nacional y Archivo Nacional— que este proyecto de ministerio no reconoce ni aprovecha.

c) En "De la estructura interna" (art. 4): El diseño actual propone dos subsecretarías (artes y patrimonio), mientras el Mineduc, con un presupuesto que supera en casi sesenta veces al del actual CNCA, tiene solo una subsecretaría.

d) En "De las subsecretarías" (arts. 8-10): Se propone una subsecretaría de las artes, con tres consejos, y en patrimonio, ninguno. Esto expresa el rasgo sectorial y productivo del diseño general del ministerio, en desmedro de áreas como, por ejemplo, el Sistema Nacional de Bibliotecas Públicas, que alcanzan hasta las regiones más remotas del país y a amplios sectores de la población. 
e) En "Establecer una vinculación permanente con el sistema educativo formal en todos sus niveles, coordinándose para ello con el Ministerio de Educación..." (art. 3): La mención es vaga y demuestra nuevamente que se concibe el Mincap de forma unívoca, sin la consideración de instrumentos y estudios para identificar las necesidades particulares de las audiencias y los espectadores. Como si la cultura fuese sólo lo que se produce para el consumo cultural o su conservación, y no un amplio espectro de manifestaciones materiales e inmateriales de las diversas comunidades. ¿Cómo asegurar una real apropiación regional de los planes y la entrega de la cultura a la cultura y de las artes a las artes? La representación civil aumenta casi en todas las instancias, sin embargo, su participación no es vinculante y la totalidad de los miembros de los consejos son elegidos por el propio ministro o de confianza de la Presidencia de la República.

f) En "De las subsecretarías regionales ministeriales" (art. 14): No se especifica relación alguna con la subsecretaría de las Artes. Sólo se menciona la del patrimonio y, de manera marginal, la relación con el propio Consejo Nacional. Esto ha sido manifestado ante las autoridades del CNCA en las últimas convenciones anuales y muestra la desarticulación del Consejo Nacional y los consejos regionales, los que no dialogan entre ellos, más allá de las instancias organizadas y presididas por funcionarios del propio CNCA o el propio ministro(a).

En este punto de la discusión, la respuesta de la institución surge del articulado del futuro ministerio: habrá consejos regionales y un consejo nacional. Tal como hay en el actual CNCA, pero hoy, al igual que mañana, la estructura misma de cada consejo regional, a pesar de denominarse como participativo, es dirigida por los subdirectores regionales $\mathrm{y}$, en el futuro, por seremis de Cultura, cargos de exclusiva confianza del Presidente(a) de la República. Es decir, el ente democrático participativo es presidido por un funcionario del mismo ministerio, dependiente del ministro(a), donde las recomendaciones, observaciones y decisiones del consejo no son vinculantes. O sea, se trata de una pantomima de la participación. Para culminar lo peculiar del diseño, es preciso recordar además que los seremis dependerán de la subsecretaría correspondiente, pero, como dice el texto, será "colaborador directo del intendente" (art. 13). Esto, además, dada la reforma que se está implementando actualmente por la que los intendentes serían elegidos demo- 
cráticamente, crearía una compleja relación triangulada entre Mincap y la autoridad regional del intendente. ${ }^{29}$

g) En "Corresponderá al Consejo Nacional" (art. 17): Aquí está la única referencia a la gestión de los fondos concursables, tema de máxima relevancia en el futuro ministerio. Estos fondos, sin considerar la creación del Fondo del Patrimonio, actualmente reparten un monto equivalente al 31,5 por ciento del presupuesto del CNCA. Sin embargo, en este punto, el proyecto se remite a la Ley 19.891, del año 2003, cuando es de público conocimiento la crisis de dichas herramientas de fomento, dadas las definiciones técnicas y sectoriales. Esto se conoce como "fondarización" del medio artístico y cultural. Estos concursos, además, están marcados por el sesgo socioeconómico y educacional de quienes los ganan, reproduciendo la brecha cultural preexistente y evidenciada en la educación. Las postulaciones a los fondos están definidas por los formularios, más que por estrategias dinámicas y actualizadas con las prácticas y procesos, de manera que ayude como herramienta de integración. Si se está diseñando una nueva institucionalidad, ¿por qué no delinear una política de fondos renovada?

Luego de esto, el resto no es más que poesía. Todo termina aquí, los fondos determinan el modelo. Es decir, lo que se está instalando es la formalización de un proyecto cultural nacional, transformado en proyecto histórico y político, por medio de un modelo económico y social puntual que se sabe obsoleto. Y dada la fuerza de los gremios - sectores productivos definidos por las tecnologías que utilizan - son estos mismos los que tienen derecho a voz, desequilibrando un escenario donde la cultura es concebida bajo un principio ontológico binario, entre los que producen cultura y las audiencias o espectadores. Parece la maqueta a escala de la operación autoficcional de la izquierda en Chile: cambiar el modelo social sin cambiar el modelo económico, —a lo que yo agregaría - pretendiendo cambiar el modelo histórico, pero no las prácticas sociales y, por lo tanto, efectivamente culturales.

h) Se menciona, por una parte, el "Consejo Nacional del Libro y la Lectura" (art. 10) como parte de la subsecretaría de las artes, cuyo objetivo es "promover proyectos, programas y acciones de apoyo a la creación literaria, la promoción de la lectura, la industria del libro, la

${ }^{29}$ Ver inciso segundo del Art. 26 de la Ley 18.575. 
difusión de la actividad literaria y el fortalecimiento de las bibliotecas públicas". Por otra parte, más adelante se dice que "se crea el Sistema Nacional de Bibliotecas Públicas” (art. 35). Esta separación orgánica puede tener graves consecuencias, ya que corresponden a subsecretarías distintas, sin asegurarse, en este proyecto de ministerio, cómo colaborarán ambas instancias de manera que no se produzca una duplicación o, peor aún, la contradicción de las políticas, sean estas anuales o quinquenales, nacionales o regionales.

i) En el artículo 2, queda expresada la jerarquía ministerial que primará: "El Ministerio, actuando como órgano rector, velará por la coordinación, consistencia y coherencia de las políticas, planes y programas en materia cultural, patrimonial, propendiendo a su incorporación en forma transversal en la actuación del Estado". No sé si es posible creer que los grandes ministerios podrán aceptar tal dependencia y, si así fuera, tampoco es posible creer que el Mincap efectivamente pueda atender todos los requerimientos de visados que aseguren la prevalencia de "las culturas, las artes y el patrimonio" en todos los casos que se requiera.

j) "Artículos transitorios". En la sección relacionada con los artículos transitorios (ver art. 4), se señala explícitamente que el Presidente de la República puede "crear, suprimir o modificar los capítulos, programas, asignaciones, ítems y glosas presupuestarias que sean pertinentes". Es decir, aplicando medidas económicas, la Presidencia puede dejar sin efecto cualquiera de las áreas existentes, las que, sin presupuesto, pueden de hecho dejar de existir. Esta capacidad presidencial se da en todos los ministerios, con la diferencia de que los otros ministerios no son participativos y en éste, en cambio, decisiones presidenciales de este tipo pueden oponerse a los acuerdos de los consejos participativos. Dado que las vías participativas de los consejos no son vinculantes, debido a la concepción jerárquica del Estado, donde no se puede contradecir al Presidente(a), me pregunto si no sería más honesto con la ciudadanía que el Mincap dejara de ser una entidad (demagógicamente) participativa y esos consejos pasaran a ser consejos o comités consultivos.

k) En esta misma sección, ver artículo 6 transitorio, "de la continuidad en los cargos por tres años de las personas que estén en las direcciones del Servicio Nacional del Patrimonio. Salvo el caso de la renuncia voluntaria". Esta mención, que está en la última página del proyecto, es la prueba de por qué hemos asistido al silencio más total 
por parte de los directivos actuales del ámbito del patrimonio. Porque de ser aprobado el proyecto, por ejemplo, se asegura la continuidad de los cargos directivos o de confianza que, dada la naturaleza de sus funciones, no debieran estar en esta sección del proyecto de ley. Es decir que, luego de la reconfirmación en sus cargos a fines del 2016 de alguno de ellos, si se aprueba el proyecto que crea el ministerio, se suman automáticamente tres años más al cargo. Prórroga inamovible sin considerar evaluación de desempeño alguna. Esta táctica prelegislativa es planteada como una forma de apoyar la transición y la adecuación hacia el ministerio, bajo la falacia de que manteniendo la jerarquía se mantiene la estabilidad.

\section{ECOS FINALES}

He participado del mundo de las artes y la cultura por más de treinta años y, aunque sé que este dato no es relevante en sí mismo, enfrentado al principio político que opera en todo proyecto de ley, creo que puedo hacerme una idea de la aplicación de la propuesta del gobierno de la Nueva Mayoría. Estamos ante un diseño inviable e impreciso, el que, como es obvio, oculta su principio más gravitante: el poder. Como es bien sabido, éste es polimorfo y da igual si está más cerca del neoliberalismo que del socialismo o del comunismo. Sé que es ingenuo de mi parte decirlo. Pero de eso es lo que efectivamente trata la ley. Lo legal de la ley. De ahí la seriedad de que, además, esto se haga por medio de la separación del Mineduc del Mincap. Se hace por algo, y para algo, algo que va más allá de lo ministerial. Es político. Sin embargo no se hace de forma explícita y con discusión pública. Eso me parece que es deshonesto con la ciudadanía y puede tener consecuencias, ahora sí, históricas. Como decía, no se apela a una discusión sobre políticas culturales, sino de sensibilidad pública en cultura, de ahí el maniqueísmo reinante.

No creo imposible aspirar a un ministerio "neutral", exento de estos sesgos que he planteado aquí; una administración que, por lo mismo, no sea en cuanto ministerio protagonista de la cultura, sino una estructura eficiente al servicio de los ciudadanos y las comunidades, entendidas en su dinamismo y equilibrio. Homeostasis cultural. Tal vez eso permitiría la acción de la cultura, el desarrollo de los quehaceres 
que fundan lo común, más allá de los formatos y productos, medios, técnicas, tecnologías o grupos y gremios que desencadenan esa experiencia imposible de separar: la de aprender, la de saber y la pertenencia a las comunidades de referencia en las que los ciudadanos viven. Por lo anterior, creo importante insistir en la falta de desarrollo de algunas áreas, así como en la inviabilidad operativa, ni siquiera ideológica, que manifiesta el proyecto en su totalidad. EP 